PERSPECTIVE

\title{
The aetiology and associations of conjunctival intraepithelial neoplasia
}

C A Kiire, B Dhillon

See end of article for authors' affiliations

.....................

Correspondence to: Dr Christine A Kiire,

Western General Hospital, Edinburgh EH4 2XU, UK; christine.kiire@doctors. org.uk

Accepted for publication 23 September 2005
Br J Ophthalmol 2006;90:109-113. doi: 10.1136/bjo.2005.077305

Aim: To summarise the main causes and associations of conjunctival intraepithelial neoplasia (CIN) found in the literature to date.

Method: Literature search using Ovid databases on the NHS Scotland E-library, Medline, and the Cochrane Library. The internet search engine Google Scholar was also used to identify relevant articles. Results: The main causes and associations of $\mathrm{CIN}$ in the literature are sun exposure and distance from the equator; human papillomavirus infection, increased p53 expression, and HIV seropositivity. It has been found that in HIV positive individuals CIN is on the increase with people being affected with more aggressive tumours at younger ages.

Conclusion: Given that $\mathrm{CIN}$ is becoming more common in HIV infected populations and that it has the potential to cause severe disability, it is important to improve our understanding of the condition. Early identification of $\mathrm{CIN}$ by an understanding of its aetiology and associations may enable the implementation of preventative measures to reduce its incidence and potentially lead to the diagnosis and further management of conditions such as HIV. Young people presenting with CIN in particular may benefit from HIV testing.

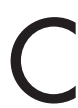
onjunctival intraepithelial neoplasia (CIN), in keeping with the general classification of other intraepithelial tumours elsewhere in the body, is dysplasia of the ocular surface epithelium that has not yet invaded the substantia propria of the conjunctiva or Bowman layer of the cornea. ${ }^{12}$ It is a subtype of ocular surface epithelial dysplasia $(\mathrm{OSED})^{3}$ or ocular surface squamous neoplasia (OSSN). ${ }^{4-6}$ OSSN is a spectrum from simple dysplasia to carcinoma in situ (CIS) to invasive squamous cell carcinoma (SCC) involving the conjunctiva as well as the cornea. Napora et $\mathrm{al}^{7}$ have included invasive SCC in their definition of conjunctival intraepithelial neoplasia. Symptoms range from none at all to severe pain and visual loss ${ }^{8}$; however, CIN and SCC of the conjunctiva commonly present with red eye and ocular irritation. ${ }^{9}$ CIN has traditionally been found at the limbus in elderly individuals. ${ }^{10}$

It is important to understand CIN because it causes disfigurement and because it can progress to SCC which has the potential to cause severe disability. ${ }^{2}$ Furthermore, it has been observed to be on the increase in certain populations. People are being affected with more aggressive tumours at younger ages. ${ }^{5}$ CIN and SCC have a high rate of recurrence. ${ }^{4}$ The cell type, clinical appearance, and degree of dysplasia do not necessarily correlate with recurrence. ${ }^{11}$ Following their investigation of the natural history of CIN, Tabin et $a l^{2}$ concluded that the slow growth of recurrent lesions combined with the potential for malignant spread was sufficient reason to suggest that all patients with a history of CIN should be followed up annually for the rest of their lives. It is crucial that we improve our understanding of this condition so that we can identify and manage it better as it becomes more common. Prevention is likely to be the key to its control.

McKelvie et al ${ }^{12}$ retrospectively studied 26 cases of SCC of the ocular surface in Melbourne, Australia, to determine pathological and clinical characteristics of tumour associated with outcome. Seven of the patients $(28 \%)$ had recurrent OSSN within 4-15 months and two of these patients died of metastatic disease. Intraocular invasion was noted in three patients, while corneal and/or scleral invasion was found in eight $(30 \%)$. Orbital invasion was noted in four patients. Six required orbital exenteration.

In the 1998 Pan American Lecture, Shields et al ${ }^{13}$ described intraocular invasion of conjunctival SCC in five patients. They found that it was more likely to occur in older patients who had one or more recurrences of a previously excised conjunctival epithelial tumour located near the corneoscleral limbus. Such patients were treated with a standard enucleation with excision of affected conjunctival issue. Metastatic disease did not develop in any of the patients. They reported that the prognosis of patients following enucleation or subtotal orbital exenteration for this condition was good.

Early identification of the aetiology and associations of CIN will enable earlier diagnosis of the condition and the implementation of preventative measures to reduce its incidence. It is likely to reduce morbidity if our understanding of the condition leads to it being diagnosed before it has a chance to develop into SCC and metastasise. Diseases thought to be associated with CIN may be picked up earlier and precautionary and therapeutic measures put into place.

Current treatments for CIN include excision and cryotherapy. ${ }^{14}$ Topical medical therapies such as mitomycin C (MMC), 5 -fluorouracil ( 5 -FU), and interferon alfa $2 \mathrm{~b}$ have also been used. MMC has been used as a both a primary treatment and an adjunct to other treatments. It is effective in the majority of cases but has been associated with complications such as ocular irritation, conjunctival hyperaemia, and punctate keratopathy. ${ }^{15}{ }^{16} 5$-FU may be a treatment option for patients with MMC resistant CINs. ${ }^{17}$ Selected cases of recurrent CIN have been successfully treated with topical and combined topical and subconjunctival interferon alfa $2 \mathrm{~b} .^{18}$ Topical interferon alfa $2 \mathrm{~b}$ as a single therapeutic agent, however, also appears to be an effective treatment that warrants further investigation in the form of large controlled studies to

Abbreviations: CIN, conjunctival intraepithelial neoplasia; CIS, carcinoma in situ; CSCN, conjunctival squamous cell neoplasia; 5-FU, 5fluorouracil; HPV, human papillomavirus; MMC, mitomycin C; OSED, ocular surface epithelial dysplasia; OSSN, ocular surface squamous neoplasia; SCC, squamous cell carcinoma 
confirm its long term efficacy and safety. ${ }^{19}$ A clear consensus on optimal management of CIN is yet to be reached. ${ }^{20}$

\section{METHOD}

The data included in this paper were collected on 20 September 2005 from a search of the Ovid databases on the NHS Scotland E-library which gives access to over 4000 journals and to databases including Medline and the Cochrane Library. The internet search engine Google Scholar was also used to identify relevant articles published in reputable books, journals and websites. The search term used was "conjunctival intraepithelial neoplasia." All articles (including case studies) describing, investigating, or reviewing the potential aetiology and associations of CIN were included. As this yielded no results from the Cochrane Library this database was searched for relevant articles using the search term "conjunctival tumour(s)." The Google Scholar search was narrowed by adding the search terms "aetiology" and "etiology".

\section{RESULTS}

The main causes and associations found for CIN are listed below:

- Sun exposure and distance from the equator

- human papillomavirus (HPV) infection

- Increased p53 expression

- HIV seropositivity

- Others.

\section{Sun exposure and distance from the equator}

Newton et $a l^{21}$ published data on the geographic distribution and incidence of SCC of the eye. They used population based cancer incidence data and published measurements of ambient solar ultraviolet light. The incidence of SCC of the eye declined by $49 \%$ for each 10 degree increase in latitude $(p<0.0001)$, falling from $>12$ cases per million per year in Uganda (latitude $0.3(0)$ ) to less than 0.2 per million in the United Kingdom (latitude $>50(0))$. Solar ultraviolet radiation decreases with increasing latitude, and the incidence of SCC of the eye decreased by $29 \%$ per unit reduction in ultraviolet exposure $(\mathrm{p}<0.0001)$. They concluded, therefore, that exposure to solar ultraviolet light is an important cause of SCC of the eye.

In 1997, Sun et al $^{22}$ described their understanding of the epidemiology of SCC of the conjunctiva in the United States in particular. They found that it was rare, with an incidence of 0.03 per 100000 but, interestingly, this rate was approximately fivefold higher among males and whites. They suspected that there may be a role of ultraviolet B radiation in the aetiology of this cancer because of the excess of SCC in tropical countries and the association between SCC of the skin and exposure to ultraviolet B. Regression analysis suggested a link between ultraviolet B exposure and prevalence of SCC of the conjunctiva. This was as strong as the link between SCC of the eyelid and ultraviolet B exposure, and much stronger than that for conjunctival melanoma and ultraviolet B exposure.

In their study of the risk factors for conjunctival squamous cell neoplasia (CSCN) (which ranged from CIN to invasive SCC) Tulvatana et $a^{23}$ investigated the role of solar elastosis. They looked at 30 consecutive pathological specimens and 30 matched controls. Elastic stain for solar elastosis was blindly interpreted in comparison with negative and positive controls. Solar elastosis was found more frequently in CSCN cases than the controls and was therefore described as a risk factor for CSCN.

Napora $\mathrm{et}^{7}$ failed to demonstrate an increased risk of CIN (ranging from dysplasia to invasive SCC by their definition) with solar exposure. Their study involved getting 19 patients with proved CIN and age and sex matched controls to complete a questionnaire to evaluate potential risk factors for CIN. They attributed this result to the small size of the study population but commented that their findings of increased risk in patients with ancestors from the British Isles, and in persons with lightly pigmented hair or eyes, may reflect increased susceptibility to ultraviolet exposure as well as genetic predisposition to development of these lesions.

\section{HPV infection}

Human papillomaviruses (HPV) are oncogenic viruses and they have been found in a variety of different epithelial neoplasias around the body-for example, cervical intraepithelial neoplasia. In an attempt to confirm the presence of human papillomaviruses in CIN, Lauer $e t a l^{24}$ studied five tumours with a polymerase chain reaction (PCR) assay designed to detect the E6 region of HPV types 16 and 18. HPV type 16 DNA was found in four of the five tumours, including two tumours that contained both type 16 and type 18 DNA. Viral DNA was not present in the fifth tumour.

The first documented cases of bilateral conjunctival tumours associated with HPV were published by Odrich et $a l^{25}$ from the Massachusetts Eye and Ear Infirmary in Boston, MA, USA. They described bilateral ocular tumours presenting as multiple keratinising and verrucous lesions of the bulbar and tarsal conjunctiva in three patients and used DNA amplification and hybridisation studies to detect HPV type 16. Two of these patients had biopsies that showed infiltrating SCC in one eye and dysplasia or carcinoma in situ in the fellow eye.

Scott et $a l^{26}$ suggested an aetiological role of HPV in CIN based on a small (10+5) study evaluating CIN and normal conjunctiva for the presence of HPV DNA and for expression (as detected by the presence of mRNA of the HPV E6 region). It was a prospective case-controlled study. The 10 cases had undergone CIN excision and there were five age matched controls (matched to five of the cases) who underwent retinal detachment repair and had no clinically identifiable conjunctival disease. HPV was detected by PCR. HPV 16 DNA and mRNA were present in five CIN specimens and HPV 18 DNA and mRNA were present in the other five specimens. Neither HPV 16 DNA/mRNA nor HPV 18 DNA/mRNA were detected in any of the control specimens or any of the clinically uninvolved conjunctival specimens $(p<0.001)$. In each of the CIN specimens, $20 \%-40 \%$ of the dysplastic cells expressed the HPV E6 region. E6 of HPV 16 and HPV 18 forms a complex with the protein encoded by p53.

Ateenyi-Agaba et $\mathrm{l}^{27}$ conducted a pilot study of epidermodysplasia verruciformis HPV types and carcinoma of the conjunctiva. They tested 21 SCC of the conjunctiva and 22 control subjects for HPV types using PCR based assays and found that epidermodysplasia verruciformis HPV types were present in $86 \%$ of SCC cases and $36 \%$ of control subjects (odds ratio -12). They suggested that there is a role of HPVs in the aetiology of SCC.

In contrast with this, Eng et $a l^{28}$ failed to detect HPV DNA in malignant epithelial neoplasms of the conjunctiva by PCR. They examined 44 formalin fixed, paraffin embedded specimens of conjunctival tumours (24 patients with papillomas and 20 patients with dysplastic and/or malignant tumours) and screened them for HPV infection using four different PCRs. They found HPV 6 and HPV 11 in nine cases of papilloma by type specific primer sets but none of the papilloma cases were positive for HPV 16 or HPV 18. They failed to demonstrate DNA of HPV 6, HPV 11, HPV 16, or HPV 18 in any of the 20 malignant epithelial tumours of the conjunctiva. Their findings of HPV 6 and HPV 11 in a significant fraction of conjunctival papillomas was in 
accordance with findings of previously reported studies. ${ }^{29}$ They concluded that malignant conjunctival carcinomas are not associated with HPV infection and posed other pathogenic mechanisms such as ultraviolet light as being more important in the aetiology of these lesions. Similarly, Tuppurainen et $a l^{30}$ failed to demonstrate any HPV DNA (types $6,11,16$, or 18) by in situ hybridisation and PCR in SCC of the conjunctiva. As SCC was so rare in Finland at that time (only four cases in the files of Kuopio University Hospital from 1959-91) their study was very limited.

\section{Increased p53 expression}

Toth $e t a^{31}$ accepted HPV as an oncogenic factor in conjunctival SCC. They conducted a study looking for a correlation between p53 overexpression and the presence of HPV infection within tumour specimens from 23 patients with conjunctival SCC. This was done because mutations of the p53 tumour suppressor gene have been found in many forms of common human cancers and because of the proposed associations between HPV and SCC of the conjunctiva. They state that the E6 protein of HPV binds with p53 and inactivates its tumour suppressor activity. They were therefore seeking an association between HPV DNA and p53 overexpression within tumour tissues. The tumour specimens were examined with light microscopy, PCR, and immunohistochemistry; $78 \%$ of the tumours were positive for p53 whereas $22 \%$ were positive for HPV. The proportion of patients positive for both p53 and HPV was $17 \%$. This was exactly the same as the proportion of patients negative for both p53 and HPV. Therefore no significant disproportion was found in the distribution of patients' HPV status and p53 status. They concluded that they were unable to show any statistical association between abnormal p53 gene product expression by immunohistochemistry in conjunctival SCC and HPV by PCR detection techniques.

\section{HIV seropositivity}

In 1995 Ateenyi-Agaba ${ }^{32}$ reported that the incidence of SCC in Kampala, Uganda, had increased from approximately 6/million/year in 1988 to 35/million/year in 1992. HIV tests were performed on all the patients $(n=48)$ who presented with conjunctival SCC between February 1990 and February 1991. $75 \%$ of these were found to be HIV seropositive compared to $19 \%$ of 48 matched controls. The relative risk for conjunctival tumours associated with HIV infection was shown to be 13.0.

Waddell et al, ${ }^{33}$ from the Uganda Eye Project, investigated the association between HIV infection and carcinoma of the conjunctiva and the role of HPV type 16. They had noticed that patients with HIV-1 seropositivity had an increased frequency of CIN and invasive SCC. They studied patients in Uganda and Malawi presenting to eye clinics with lesions suspicious of carcinoma. The study in Uganda was a casecontrol study and in Malawi HIV data were collected on consecutive presenting patients. For the study in Uganda there were two controls per case, matched for age and sex, and they came from the same health unit or lived in the same district. Pathological confirmation of eye lesions was sought. HIV testing was performed on patients who were biopsied, and, in Uganda, matched control subjects. A sample of fixed biopsies was tested for HPV 16 by PCR. The HIV-1 serology, histopathology of conjunctival biopsies, and prevalence of HPV infection were determined. Carcinoma was significantly associated with HIV infection in Ugandan patients with an odds ratio of 13.1 (95\% confidence interval 4.7 to 37.6 ). In Malawi there were no controls from which to calculate an odds ratio but $78 \%$ of the cases were HIV positive (compared to $33 \%$ HIV-l seroprevalence in antenatal women in the same hospital).
Karp et $a l^{10}$ studied records of patients at the Bascom Palmer Eye Institute (Miami, FL, USA) in whom CIN was diagnosed between 1991 and 1993. They were particularly interested in checking the HIV status of the patients under the age of 50. They were able to contact six out of nine of these and found that three $(50 \%)$ were HIV positive. It was, therefore, concluded that HIV testing and counselling should be considered in patients younger than 50 years in whom CIN is diagnosed.

The clinical characteristics of SCC and CIN in AIDS patients were studied further by Kaimbo Wa Kaimbo et al ${ }^{34}$ in the Congo. They reviewed biopsy results of patients with confirmed SCC and CIN between 1994 and 1997. Three of these had SCC and seven had CIN. They found that SCC and CIN in their patient group had similar clinical characteristics as immunocompetent people but they were occurring at a younger age and were more aggressive.

Poole $^{35}$ assessed the increase in incidence of conjunctival SCC over a 22 year period in Tanzania in an attempt to analyse possible reasons for change. It was a retrospective analysis of records from a Tanzanian pathology department serving north and central Tanzania from 1976 to 1997. He looked at medical records of patients diagnosed with conjunctival SCC in the past 2 years of the study. A sharp rise in the incidence of conjunctival SCC in the past 3 years of the study was found. It was suspected that this epidemic could be related to HIV infection on a background of ultraviolet light exposure. Only five patients had been tested for HIV status and four of these were positive.

Porges and Groisman ${ }^{3}$ assessed the link between SCC/CIS and HIV. They conducted a case-control study in an African provincial hospital. Twenty three black African patients underwent excisional biopsy of conjunctival malignant lesions; 18 of these agreed to have an ELISA test for HIV antibodies before the excisional biopsy. Twelve of the patients had SCC, six had CIS, and five had Kaposi's sarcoma. There were seven controls with benign conjunctival lesions. Seropositivity for HIV was found to be significantly higher in the SCC/CIS group than in the control group. The most common clinical finding in the SCC/CIS/HIV group (12 patients) was corneal overriding. This was found in 11 of the 12 patients. Conjunctival malignancy was the first presenting sign for AIDS in 50\% of their patients.

The association of malignant tumours of the conjunctiva and HIV infection in Kinshasa (Democratic Republic of Congo) was also studied by Timm et al. ${ }^{36}$ They examined histologically 34 specimens of conjunctival tumours removed at the University of Kinshasa in 2001 and tested all the patients concerned for HIV infection; 18 out of 34 patients were HIV positive. The results showed that there was no difference in the frequency of CIN between HIV seropositive and HIV seronegative patients. They did note, however, that HIV seropositive patients with a SCC were on average 17 years younger than those who were HIV seronegative.

Two recent case reports of CIN and SCC respectively have been identified in the literature. Cackett $e t$ a ${ }^{37}$ described a case of a 38 year old Zambian woman with a conjunctival lesion that predated her diagnosis of HIV by 5 years. It had initially been thought to be benign and as such had been managed conservatively. When a diagnosis of HIV seropositivity was made the lesion was biopsied and found to be CIN. De Silva $e t a l^{38}$ described a case of a Nigerian patient with a right lower lid swelling that was initially treated as a chalazion but was found, 5 months later, to be widespread metastatic SCC associated with positive HIV serology.

\section{Others}

Exposure to petroleum products, heavy cigarette smoking, light hair, ocular pigmentation, family origin the United 
Kingdom, Austria, or Switzerland, non-office and nonprofessional workers, and those who are not college graduates have all been thought to be factors associated with CIN. ${ }^{7}$ These data were obtained by asking 19 patients with biopsy proved CIN to complete a questionnaire to evaluate possible predisposing factors.

In their case-control study of conjunctival SCC in adults presenting at hospitals in Kampala, Uganda, Newton et al ${ }^{39}$ interviewed participants about social and lifestyle factors. In addition, they tested their blood for antibodies to HIV, KSHV, and HPV 16, HPV 18, and HPV 45. The odds of each factor occurring in 60 people with conjunctival cancer was compared to that in 1214 controls with other cancer sites of types using odds ratios estimated with unconditional logistic regression. The study showed that conjunctival cancer was associated with HIV infection (10-fold increased risk of conjunctival cancer in HIV infected individuals-similar to other studies) and was less common in those with a higher personal income. The risk of conjunctival cancer increased with increasing time spent in cultivation and therefore in direct sunlight, but decreased with decreasing age at leaving home, thought possibly to reflect less exposure to sunlight as a result of working in towns. Both of these results were of borderline significance. Sexual and reproductive variables were examined among HIV seropositive individuals. Cases were more likely than controls to report that they had given or received gifts for sex (OR 3.5, p = 0.03), but this may have been a chance finding as no other sexual or reproductive variable was associated with conjunctival cancer, including the number of self reported lifetime sexual partners. The seroprevalence of antibodies against HPV 18 and HPV 45 was too low to make reliable conclusions. The presence of antiHPV antibodies was not significantly associated with SCC and nor were anti-KSHV antibodies.

Whittaker et $a l^{40}$ described a case of leucoplakia and underlying intraepithelial squamous cell neoplasia of the palpebral conjunctiva presenting in a 62 year old man wearing an ocular prosthesis. He was successfully treated with tumour excision and lid reconstruction. They recommended that the sockets of patients wearing long standing ocular prostheses are examined regularly in order to avoid missing such lesions.

Contact lens wear has also been implicated in the pathogenesis of ocular surface epithelial dysplasia. ${ }^{41}$

\section{DISCUSSION}

There are problems with many of the studies investigating the possible causes of CIN. They vary significantly in terms of study design, quality, consistency and directness.

A small number of eyes have been examined in the majority of the studies. This could be because this condition is rare, and it was very rare until recently. Most of the researchers have attempted to match cases and controls in terms of age and sex in an attempt to reduce the numbers of confounding factors and gain results with the highest statistical significance. As Napora et $a l^{7}$ explained, case-control studies permit the investigation of multiple exposures and are therefore appropriate for the study of relatively rare diseases.

The data on sun exposure are largely based on studies of SCC. Newton et $a l^{21}$ showed evidence of a dose-response gradient. There are, however, likely to be multiple confounding factors in their interpretation of the difference in incidence of SCC in Uganda and the United Kingdom. They have attributed this huge difference $(>12$ cases per million in Uganda $v<0.2$ cases per million in the United Kingdom) to the difference in solar ultraviolet light but it is clear from the findings about HIV and CIN and/or SCC in Uganda that it is unlikely to be as straightforward as this. The regression analysis by Sun et al $^{22}$ suggesting a link between ultraviolet $\mathrm{B}$ exposure and prevalence of SCC is not specific enough to be interpreted as evidence of a significant link between ultraviolet B and CIN.

Tulvatana $e^{2}$ al $^{23}$ did not differentiate between CIN and SCC in their investigation of the role of solar elastosis in what they called conjunctival squamous cell neoplasia (CSCN). It may therefore not be possible to extrapolate their findings of solar elastosis as a risk factor for CSCN to specifically cover CIN, even though their study was a case-control study involving a relatively large number of specimens and controls.

When assessed according to guidelines provided by the GRADE Working Group, ${ }^{42}$ Lauer et al's work ${ }^{24}$ is moderately suggestive of a link between HPV 16 and CIN and less suggestive of a similar link between HPV 18 and CIN. The low number of tumours studied, however, significantly weakens the strength of the evidence. The prospective case-controlled study by Scott et $a^{26}$ had slightly more patients and controls. It adds to the body of moderate grade evidence suggestive of a link between HPV 16, HPV 18, and CIN.

Ateenyi-Agaba ${ }^{27}$ studied HPV and SCC, but not CIN, and found very strong evidence of a link between the two. This distinctly conflicted with findings by Eng $e a^{28}$ that there was no association between HPV infection and malignant conjunctival carcinomas. Tuppurainen et al's work ${ }^{30}$ supports that of Eng et al, ${ }^{28}$ but is weak evidence as only four cases were identified and investigated.

The data on increased p53 expression ${ }^{31}$ were partially limited by the acceptance of HPV as an oncogenic factor in SCC. As it was not specifically looking at CIN our ability to assume that there is no association between increased p53 expression and CIN is limited.

The evidence provided by Ateenyi-Agaba ${ }^{32}$ and Poole ${ }^{35}$ for a link between HIV seropositivity and SCC is very strong. It must be noted, however, that like many of the studies already discussed it is not clear how easily one can extrapolate these results to CIN. Waddell $e t$ al $^{33}$ differentiated between CIN and SCC in their investigation of the link between HIV-1 seropositivity and these tumours. Their evidence, particularly from the Uganda Eye Project, is equally impressive. The work they did in Malawi, ${ }^{33}$ and that of Karp et al, ${ }^{10}$ supports the findings above.

Kaimbo Wa Kaimbo's study of CIN and SCC (clearly differentiated) in the Congo in $1998^{34}$ provides us with one of the most significant reasons to be aware of the potential aetiology and associations of CIN-the risk of more aggressive tumours occurring in younger patients with AIDS. The number of biopsies reviewed was small (10) but the potential significance of their findings makes this an area worth investigating further. Following the finding by Porges and Groisman ${ }^{3}$ that conjunctival malignancy was the first presenting sign for AIDS in $50 \%$ of their patients it was suggested that an HIV test be performed in cases of conjunctival SCC/CIS or dysplasia, especially in patients in high risk populations.

The study by Timm et $a^{36}$ differentiates between CIN, SCC, and other diseases of the conjunctiva. It helpfully considers the frequency of CIN in patients with and without HIV seropositivity and finds that there is no difference. The finding of SCC occurring at a younger age in HIV seropositive patients is consistent with the findings of Kaimbo Wa Kaimbo (also based in the Congo). ${ }^{34}$ The number of patients investigated was relatively good at 34 , with all patients being tested for HIV.

The authors of the two case reports urged that HIV be considered in young patients with atypical conjunctival lesions and that young patients from countries with a high HIV prevalence (and high ultraviolet B radiation exposure) be given a low threshold for excision biopsy of conjunctival lesions. ${ }^{37} 38$

If HIV is, as the majority of the above studies suggest, an infection that predisposes one to CIN then this is a very 
significant confounding factor in the studies carried out in countries where other variables were being investigated but in which HIV is very common. It would be interesting to know if cervical CIN was elevated in HIV to establish the significance of the part played by solar elastosis in the aetiology of CIN.

Assessment of evidence via questionnaires yields a low grade of evidence as defined by the GRADE Working Group. ${ }^{42}$ It is, however, the main method with which the association between social and lifestyle factors and CIN and SCC have been assessed. It is therefore not surprising that there are wide discrepancies in the descriptions of the different types of people thought to be at risk of these tumours. ${ }^{7239}$ The evidence found implicating ocular prostheses and contact lens wear is anecdotal..$^{41}$

In summary, sun exposure, HPV infection and HIV infection seem to be the factors most likely to cause or be associated with CIN. The overlap of these factors in the parts of the world where the incidence of CIN is rapidly rising implies that they probably interact and that no one factor on its own can be described as causing CIN.

So what can be done with the information available to us so far? Should we be doing everything we can to protect our eyes from the sun? More importantly should everyone found to have CIN be offered an HIV test? Perhaps this should only occur in areas where HIV is highly prevalent. Should only the young patients presenting with aggressive tumours have HIV tests or are they worth considering in all cases?

\section{CONCLUSION}

In conclusion, conjunctival CIN is an important condition to know about because it is becoming more common, may be a marker for HIV, is disfiguring, and may progress to SCC with its potential to cause disability and metastasise. It is likely to increase the burden of disease in those countries with the most limited resources. We believe that the most important causes and associations of CIN are likely to be sun exposure, HPV and HIV. Of these, it is our opinion that it would be of greatest value to further understand the links between HIV and CIN because of the potential to intervene early to potentially reduce to spread of HIV by informing patients of their diagnosis possibly earlier than would otherwise have been the case and to reduce morbidity in people who are already immunodeficient. Practical and inexpensive ways of preventing the condition and treating it early will have to be identified if the numbers of people affected are going to increase.

\section{Authors' affiliations}

C A Kiire, Western General Hospital, Edinburgh EH4 2XU, UK B Dhillon, Princess Alexandra Eye Pavilion, Edinburgh EH3 9HA, UK

Competing interests: Nil.

\section{REFERENCES}

1 Pizzarello LD, Jakobiec FA. Bowen's disease of the conjunctiva: a misnomer. In Jakobiec FA, eds. Ocular and adnexal tumours. Birmingham: Aesculapius, 1978:553-71.

2 Tabin G, Levin S, Snibson G, et al. Late recurrences and the necessity for longterm follow up in corneal and conjunctival intraepithelial neoplasia. Ophthalmology 1997; 104:485-92.

3 Porges Y, Groisman. Prevalence of HIV with conjunctival squamous cell neoplasia in an African provincial hospital. Cornea 2003;22:1-4.

4 Lee GA, Hirst LW. Retrospective study of ocular surface squamous neoplasia. Aust N Z J Ophthalmol 1997;25:269-76.

5 Pola EC, Masanganise R, Rusakaniko S. The trend of ocular surface squamous neoplasia among ocular surface tumour biopsies submitted for histology from Sekuru Kaguvi Eye Unit, Harare between 1996 and 2000. Central African J Med 2003;49:1-4.

6 Basti S, Macsai M. Ocular surface squamous neoplasia: a review. Cornea 2003;22:687-704.

7 Napora C, Cohen EJ, Genvert Gl, et al. Factors associated with conjunctival intraepithelial neoplasia: a case control study. Ophthalmic Surg 1990;21:27-30.
8 Lee GA, Hirst LW. Ocular surface squamous neoplasia. Surv Ophthalmol 1995; 39:429-50.

9 Tunc M, Char DH, Crawford B, et al. Intraepithelial and invasive squamous cell carcinoma of the conjunctiva: analysis of 60 cases. $\mathrm{Br} J$ Ophthamol 1999;83:98-103.

10 Karp CL, Scott IU, Chang TS, et al. Conjunctival intraepithelial neoplasia. A possible marker for human immunodeficiency virus infection? Arch Ophthalmol 1996;114:257-61.

11 Erie JC, Campbell J, Liesegang TJ. Conjunctival and corneal intraepithelial and invasive neoplasia. Ophthalmology 1986;93:176-83.

12 McKelvie PA, Daniell M, McNab A, et al. Squamous cell carcinoma of the conjunctiva: a series of 26 cases. Br J Ophthalmol 2002;86:168-73.

13 Shields JA, Shields CL, Gunduz K, et al. The 1998 Pan American Lecture. Intraocular invasion of conjunctival squamous cell carcinoma in five patients. Ophthalmic Plast Reconstruct Surg 1999;15:153-60.

14 Peksavar G, Altan-Yaycioglu R, Onal S. Excision and cryosurgery in the treatment of conjunctival malignant epithelial tumours. Eye 2003;17:228-32.

15 Rozenman Y, Frucht-Pery J. Treatment of conjunctival intraepithelial neoplasia with topical drops of mitomycin C. Cornea 2000;19:1-6.

16 Prabhasawat P, Tarinvorakup P, Tesavibul N, et al. Topical $0.002 \%$ mitomycin $C$ for the treatment of conjunctival-corneal intraepithelial neoplasia and squamous cell carcinoma. Cornea 2005;24:443-8.

17 Yamamoto N, Ohmura T, Suzuki H, et al. Successful treatment with 5fluorouracil of conjunctival intraepithelial neoplasia refractive to mitomycin-C. Ophthalmology 2002;109:249-52.

18 Toledeno Fernandez N, Garcia Saenz S, Diaz Valle D, et al. Interferon alfa$2 \mathrm{~b}$ treatment in selected cases of recurrent conjunctival intraepithelial neoplasia. [Spanish]. Archivos de la Sociedad Espanola de Oftalmologia 2003;78:265-71

19 Boehm MD, Huang AJ. Treatment of recurrent cornea and conjunctival intraepithelial neoplasia with topical interferon alfa $2 \mathrm{~b}$. Ophthalmology 2004;111:1755-61.

20 Stone D, Butt A, Chodosh J. Ocular surface squamous neoplasia: a standard of care survey. Cornea 2005;24:297-300.

21 Newton R, Ferlay J, Reeves G, et al. Effect of ambient solar ultraviolet radiation on incidence of squamous-cell carcinoma of the eye. Lancet 1996;347:1450-1.

22 Sun EC, Fears TR, Goedert JJ. Epidemiology of squamous cell conjunctival cancer. Cancer Epidemiol Biomarkers Prev 1997;6:73-7.

23 Tulvatana W, Bhattarakosol P, Sansopha I, et al. Risk factors for conjunctival squamous cell neoplasia: a matched case-control study. Br J Ophthalmol 2003;87:396-8.

24 Laver SA, Malter JS, Meier JR. Human papillomavirus type 18 in conjunctival intraepithelial neoplasia. Am J Ophthalmol 1990;1 10:23-7.

25 Odrich MG, Jakobiec FA, Lancaster WD, et al. A spectrum of bilateral squamous cell conjunctival tumours associated with human papillomavirus type 16. Ophthalmology 1991;98:628-35.

26 Scott IU, Karp CL, Nuovo GJ. Human papillomavirus 16 and 18 expression in conjunctival intraepithelial neoplasia. Ophthalmology 2002;109:542-7.

27 Ateenyi-Agaba C, Weiderpass E, Smet A, et al. Epidermodysplasia verruciformis human papillomavirus types and carcinoma of the conjunctiva: a pilot study. Br J Cancer 2004;90:1777-9.

28 Eng HL, Lin TM, Chen SY, et al. Failure to detect human papillomavirus DNA in malignant epithelial neoplasms of conjunctiva by polymerase chain reaction. Am J Clin Pathol 2002;117:429-36.

29 Sjo NC, Heegaard S, Prause JU, et al. Human papillomavirus in conjunctival papilloma. Br J Ophthalmol 2001;85:785-7.

30 Tuppurainen K, Raninen A, Kosunen O, et al. Squamous cell carcinoma of the conjunctiva. Failure to demonstrate HPV DNA by in situ hybridization and polymerase chain reaction. Acta Ophthalmol 1992;70:248-54.

31 Toth J, Karcioglu ZA, Moshfeghi AA, et al. The relationship between human papillomavirus and p53 gene in conjunctival squamous cell carcinoma. Cornea 2000;19:159-62.

32 Ateenyi-Agaba C. Conjunctival squamous-cell carcinoma associated with HIV infection in Kampala, Uganda. Lancet 1995;345:695-6.

33 Waddell KM, Lewallen S, Lucas SB, et al. Carcinoma of the conjunctival and HIV infection in Uganda and Malawi. Br J Ophthalmol 1996:80:503-8.

34 Kaimbo Wa Kaimbo D, Parys-Van Ginderdeuren R, Missotten L. Conjunctival squamous cell carcinoma and intraepithelial neoplasia in AIDS patients in Congo Kinshasa. Bull Soc Belge Ophtalmol 1998;268:135-41.

35 Poole TR. Conjunctival squamous cell carcinoma in Tanzania. Br J Ophthalmol 1999;83:177-9.

36 Timm A, Stropahl G, Schittowski M, et al. Association of malignant tumours of the conjunctiva and HIV infection in Kinshasa (D R Congo). First results. [German]. Ophthalmologe 2004;101:1011-16.

37 Cackett $\mathbf{P}$, Gillies $M$, Leen $C$, et al. Conjunctival intraepithelial neoplasia in association with HIV infection. AIDS 2005;19:351-2.

38 De Silva DJ, Tumuluri K, Joshi N. Conjunctival squamous cell carcinoma: atypical presentation of HIV. Clin Experiment Ophthalmol 2005;33:419-20.

39 Newton R. A review of the aetiology of squamous cell carcinoma of the conjunctiva. Br J Cancer 1996:74:1511-13.

40 Whittaker KW, Trivedi D, Bridger J, et al. Ocular surface squamous neoplasia: report of an unusual case and review of the literature. Orbit 2002;21:209-15.

41 Guex-Crosier Y, Hervot C. Presumed corneal intraepithelial neoplasia associated with contact lens wear and intensive ultraviolet light. Br J Ophthalmol 1993;77:191-2.

42 GRADE Working Group. Grading quality of evidence and strength of recommendations. BMJ 2004;328:1490. 